\title{
The Experience of Using "Neovasculgen" in the Complex Treatment of Patients with Critical Lower Limb Ischemia
}

\author{
Mikhail M. Vinokurov, $\mathrm{PhD}, \mathrm{ScD}^{1 *}$; Anton A. Yakovlev, $\mathrm{PhD}^{2}$; Innokenty D. Ushnitsky, \\ $\mathrm{PhD}, \mathrm{ScD}^{1}$; Gennady A. Palshin, $\mathrm{PhD}, \mathrm{ScD}^{1}$; Aleksander P. Petrov, $\mathrm{PhD}^{2}$; \\ Vladimir V. Popov²; Tatyana V. Yalinskaya² \\ ${ }^{1} M$. K. Ammosov North-Eastern Federal University \\ ${ }^{2}$ Republican Hospital No. 2-Center for Emergency Medical Care \\ Yakutsk, the Republic of Sakha (Yakutia), Russia
}

\begin{abstract}
This article presents an assessment of the clinical efficacy and long-term results of the use of pl-VEGF165 (a gene therapy drug Neovasculgen) in the treatment of patients with critical lower limb ischemia. The present study revealed a significant decrease in the degree of ischemia, pain reduction and relief; an increase in pain-free walking distance; a regression of trophic disorder; a decrease in the number of amputations; and an increase in the number of lower limb preservations.(International Journal of Biomedicine. 2020;10(3):287-290.)
\end{abstract}

Key Words: chronic lower limb ischemia $\bullet$ lower extremity arteries $\bullet$ pain-free walking distance $\bullet$ linear blood flow velocity

\section{Abbreviations}

ATA, anterior tibial artery; BF, blood flow; CDUS, color Doppler ultrasonography; CFA, common femoral artery; ChLLI, chronic lower limb ischemia; CrLLI, critical lower limb ischemia; DPA, dorsalis pedis artery; LBFV, linear blood flow velocity; LEA, lower extremity arteries; LL, lower limb; PTA, posterior tibial artery; PFA, profunda femoris artery [deep femoral artery]; PA, popliteal artery; PWD, pain-free walking distance; SFA, superficial femoral artery.

\section{Introduction}

Currently, chronic lower limb ischemia (ChLLI) remains an urgent problem of angiology and vascular surgery due to the difficulty and duration of treatment, and the high prevalence and high degree of disability and mortality of patients. ${ }^{(1,2)}$ More than half of patients with peripheral artery disease at the time of treatment already present stage IIb (pain-free walking distance [PWD] of $<200 \mathrm{~m}$ ) according to the Fontaine-Pokrovsky classification. ${ }^{(3)}$ Such patients are candidates for endovascular open or hybrid surgical treatment. ${ }^{(4)}$ Nevertheless, the high level of modern reconstructive surgery of the lower extremity

*Corresponding author: Prof. Mikhail M. Vinokurov, MD, PhD, ScD. M. K. Ammosov North-Eastern Federal University. Yakutsk, the Sakha Republic, Russia. E-mail: mmv_mi@rambler.ru arteries (LEA) cannot solve all the problems in this group of patients. The success of reconstructive interventions directly depends on the condition of the so-called outflow pathwaysvessels located below the inguinal fold. ${ }^{(5)}$ According to some reports, up to $40 \%$ of patients requiring surgical treatment cannot undergo arterial reconstruction due to distal- (or) widespread multifocal lesions of the arterial bed.

Modern reconstructive vascular surgery and angiotropic pharmacotherapy can achieve positive results in a large number of patients. However, there are limitations on the possibilities of existing treatment approaches in relation to patients with diffuse lesions of the distal arterial bed, for which the longterm results of surgical treatment are unsatisfactory, and pharmacotherapy is effective only in the early stages of the disease. This category of patients makes the main contribution to the unsatisfactory results of ChLLI treatment. ${ }^{(3,4,6)}$ Currently, of modern principles of action, the use of pl-VEGF 165 
(Neovasculgen) is promising for the further study and use of new approaches in the treatment of ChLLI patients.

Neovasculgen is a first-in-class gene therapy drug developed by the Human Stem Cells Institute and approved in 2011 for human use in Russia for treatment of atherosclerotic $\mathrm{PAD}$, including critical limb ischemia.(7) This drug is an original gene construction in which pl-VEGF165 $1.2 \mathrm{mg}$ is the active substance, whose mechanism, therapeutic angiogenesis, introduces a new approach to treating ischemia: the use of an evolutionarily programmed process of blood vessel creation and growth. ${ }^{(8,9)}$

The aim of this work was to evaluate the clinical efficacy and long-term results of the use of pl-VEGF165 (a gene therapy drug Neovasculgen) in the treatment of patients with ChLLI.

\section{Materials and Methods}

In total, 23 patients $(15 / 65.2 \%$ men and $8 / 34.8 \%)$ with ChLLI that correlated with stage II-IV, according to the Fontaine-Pokrovsky classification (2004), were included in the study in the period from 2015 to 2019. Conditions for inclusion in the study were the presence of multistory lesions of the vascular bed, the absence of a good distal vascular bed, and distal arteries of the lower extremities on which reconstructive interventions could not be performed. Atherosclerotic genesis of ChLLI was determined in $16(69 \%)$ cases, diabetes mellitus in $5(21 \%)$ cases, and thromboangiitis obliterans with necrotic changes in the toes in $2(8.6 \%)$ cases. The age of the patients ranged from 22 to 76 years(average age of 66 years).

All patients were divided into 3 groups according to the Fontaine-Pokrovsky classification: Group 1 included 8(34.8\%) patients with stage IIa (PWD from $200 \mathrm{~m}$ to $500 \mathrm{~m}$ ); Group 2 included 10(43.5\%) patients with stage IIb (PWD of $<200 \mathrm{~m}$ but $>50 \mathrm{~m})$; Group 3 included $5(21.7 \%)$ patients with stage III-IV (PWD of $\leq 50 \mathrm{~m}$ with trophic and necrotic changes in the toes).

All patients suffered from pain syndrome of various intensity and $15(65 \%)$ had a constant pain in the foot. Two $(8.6 \%)$ patients had a history of open reconstructive vascular surgeries.

All patients underwent angiography of LEA at the beginning and after the course of treatment. CDUS (HI VISION Preirus) of the LEA was performed at the beginning of treatment, during treatment and at the end of the of treatment course. The level of PWD was determined by the treadmill test. The stage of limb ischemia was evaluated before and after treatment. All patients received standard conservative therapy for ChLLI without cilostazol and prostaglandins. Neovasculgen was administered into calf muscles twice, with an interval of 14 days between them, at a dose of $1.2 \mathrm{mg}$ dissolved in $2 \mathrm{ml}$ of water for injections at 4 to 5 injection sites located as close as possible to the ischemic area, according to the standard scheme ${ }^{(3)}$ After ending the course of treatment, control examination was performed in the period from 3 months to 4 years.

The effectiveness of therapy was evaluated as follows: -A significant improvement - a decrease in the stage of ischemia according to the Fontaine-Pokrovsky classification -A moderate improvement and preservation of the previous stage of ischemia with an increase in PWD from the initial values

-A satisfactory result and stabilization of ChLLI without a significant increase in PWD; a regression of necrotic processes without surgical interventions, and after surgical treatment at stage IIIb

-A deterioration and increase in the stage of ischemia, the transition of ChLLI to CrLLI, amputation of a limb, death from any cause.

When evaluating clinical efficacy, a clear positive dynamics was established for all groups of patients. In Group 1 , PWD increased in all patients and amounted to $200 \pm 150 \mathrm{~m}$; pain relief was noted. In Group 2, 8 out of 10 patients moved from ChLLI stage IIb to stage IIa with PWD of $213 \pm 209 \mathrm{~m}$. In Group 3, 2 patients moved from stage III to stage IIa; in 2 patients we noted the transition from ChLLI to CrLLI with LL amputation in the second and third months after Neovasculgen therapy (Fig.1.).

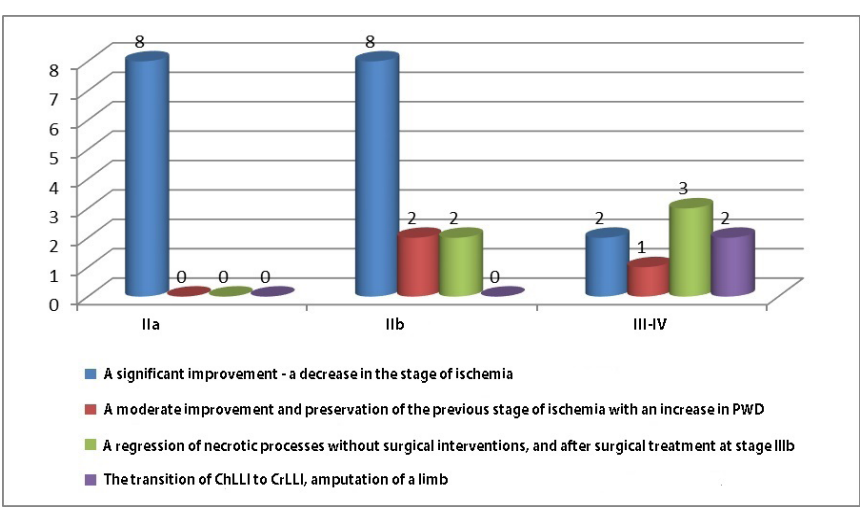

Fig.1. The clinical efficacy of the use of Neovasculgen

For patients with diabetes mellitus, satisfactory results were achieved in 4 cases; one case ended with LL amputation. In remote observations, the best results in improving the quality of life were obtained in patients with stages IIa and IIb.

\section{Clinical examples of the efficacv of Neovasculgen}

\section{Case report 1}

A 24-year-old white women with a history of smoking (more than 2 packs per day) presented to the surgical department of Republican Hospital No. 2 with a complaint of periodically occurring rest pain in the right foot with weak relief after analgesics, and trophic changes in the fourth toe of the right foot with the transition of skin necrosis to the foot. CDUS and angiography of LEA, as well as clinical and laboratory examination, were performed. It was not possible to evaluate the PWD, as the patient could not step on the LL due to trophic lesions of the foot.

The results of angiography revealed a distal type of lesion of the vascular system of LL. We decided to refuse performing an open reconstructive operation due to the nonoperability state of the patient. An attempt at endovascular balloon angioplasty was without a positive result. 
CDUS of LEA before (1) and after (2) a double injection of Neovasculgen (one month after of beginning treatment)

1. CDUS (01/09/2019).

Right LL: CFA, DPA, PA, and SFA are passable and accessible throughout the entire study, their gaps in the visible segments were free, and the intima-media complex with preserved differentiation into layers was not thickened. Stenoses were not detected. BF throughout the arterial bed was symmetrical, of the main type, with the usual speed and spectral characteristics.

PTA: the upper third - a collateral type of BF; the middle third - multiple crimped collaterals, a reduced BF, LBFV of up to $25 \mathrm{~cm} / \mathrm{s}$; the lower third - the tortuous collaterals, BF is not recorded. ATA: the upper third - LBFV of up to $60 \mathrm{~cm} / \mathrm{s}$; the middle third - LBFV of up to $40 \mathrm{~cm} / \mathrm{s}$; the lower third - LBFV of up to $25 \mathrm{~cm} / \mathrm{s}$, multiple crimped collaterals.

2. CDUS (02/05/2019).

Right LL: CFA, DPA, PA, and SFA are of normal echogenicity, passable, and with thin walls; $\mathrm{BF}$ of magistral type. PTA: a collateral type of BF; LBFV of up to $20-24 \mathrm{~cm} / \mathrm{s}$. ATA: no stenoses; LBFV of up to $55 \mathrm{~cm} / \mathrm{s}$. DPA: a reduced BF, LBFV of up to $15-27 \mathrm{~cm} / \mathrm{s}$.. Multiple collaterals were visualized along the front surface of the foot.

Left LL: CFA, DPA, PA, and SFA are of normal echogenicity, passable, and with thin walls; BF of magistral type. ATA was passable, of small diameter with thin walls; a reduced $\mathrm{BF}$ with LBFV of up to $25 \mathrm{~cm} / \mathrm{s}$, which was decreased distally up to $15 \mathrm{~cm} / \mathrm{s}$; BF of a collateral type. DPA: a magistral-altered type of BF, LBFV of up to $22 \mathrm{~cm} / \mathrm{s}$. PTA: uneven narrowing, uneven course of the vessel, a collateral type of BF; LBFV decreased up to $25 \mathrm{~cm} / \mathrm{s}$.

As a result of treatment, there were positive dynamics in the indicators of micro- and macrocirculation, according to CDUS, trophic change regression in the toe, the absence of rest pain, and an increase in PWD more than $100 \mathrm{~m}$. Further treatment was continued. After 2 months, necroectomy of the necrotic toe was performed with healing by primary intention. Thus, the result of the treatment was LL preservation and an increase in PWD level.

\section{Case report 2}

A 56-year-old white male suffering from Type 2 diabetes mellitus, in 2014 for the first time noted the appearance of pain in the calf muscles, mainly on the right, when walking a distance of $300 \mathrm{~m}$. As time went on, the disease progressed. PWD decreased down to $100 \mathrm{~m}$; necrotic changes of the fourth and fifth toes of the right foot appeared, with rest pain. CDUS and angiography of LEA, as well as clinical and laboratory examination, were performed.

According to the results of angiography, stenosing atherosclerotic plaques (small, medium and high gradations of narrowing) of the LEA were revealed, as well as an occlusion in the lower third of SFA on the right, arterial stenosis of SFA (high and critical gradations with hemodynamic disturbances) on the left, stenosis of moderate gradations on both sides, small to moderate stenosis of ATA on both sides, and an occlusion of PTA in the upper and middle thirds on both sides. Inadequate development of collaterals was also noted.
CDUS of LEA before (1) and after (2) a double injection of Neovasculgen ( 26 days after of beginning treatment)

1. CDUS (01/13/2015).

Right LL: CFA was passable, BF of the magistral type, stenosis of small gradations (up to 30\%) of the artery mouth at the level of bifurcation. PFA was passable, stenosis of small gradations, the magistral type of BF. SFA was passable at the level of the upper third, the altered BF; at the level of the middle third of the thigh, heterogeneous thrombotic masses completely obstructing the artery mouth for approximately $15-20 \mathrm{~cm}$; BF was not visualized. At the level of the lower third of the thigh (at the SFA mouth), the blood flow was collateral, LBFV of up to $35-29 \mathrm{~cm} / \mathrm{s}$. PA was passable, moderate stenosis, LBFV of up to $35 \mathrm{~cm} / \mathrm{s}$, a reduced collateral type of BF. ATA was passable, $\mathrm{BF}$ of the magistral type, a reduced BF, LBFV of up to 25-30 $\mathrm{cm} / \mathrm{s}$. PTA: occlusions at the upper/middle thirds; at the level of the lower third - collateral inflow with LBFV of up to $17 \mathrm{~cm} / \mathrm{s}$. DPA was passable; a reduced and collateral BF.

Left LL: CFA was passable, BF of the magistral type, stenosis of small gradations (up to $30 \%$ ) of the mouth at the level of bifurcation. PFA was passable, LBFV of up to 80$89 \mathrm{~cm} / \mathrm{s}$, a magistral-altered BF type. SFA was passable, a pronounced stenosis at the upper third and moderate stenosis at the middle/lower thirds, and developed collateral BF networks. PA was passable, moderate stenosis, a reduced BF, LBFV of up to $58 \mathrm{~cm} / \mathrm{s}$, a magistral-altered BF type. ATA was passable, a moderate stenosis, LBFV of up to $70 \mathrm{~cm} / \mathrm{s}$, a magistralaltered BF type. PTA: occlusions at the upper/middle thirds; a collateral inflow at the level of the lower third; LBFV of up to $44 \mathrm{~cm} / \mathrm{s}$.

\section{2. $\operatorname{CDUS}(02 / 07 / 2015)$.}

Left LL: CFA was passable, stenosis of $30 \%-40 \%$, an unimpaired BF. PFA was passable, stenosis of $30 \%-40 \%$, an unimpaired BF. SFA was passable, stenosis of $20 \%-30 \%$, an unimpaired BF. PA was passable, stenosis of $30 \%$, an unimpaired BF. ATA and DPA were passable, a moderate stenosis. PTA was passable, a high stenosis, a reduced collateral type BF; LBFV of up to $26 \mathrm{~cm} / \mathrm{s}$.

Right LL: CFA was passable, stenosis of $30 \%-40 \%$, an unimpaired BF. PFA was passable, stenosis of $30 \%-40 \%$, an unimpaired BF. SFA was passable, stenoses at the upper/ middle thirds up to $65 \%-75 \%$ and $30 \%$ at the lower third; a magistral-altered reduced $\mathrm{BF}$, starting from the upper third and distally, LBFV of up to $40 \mathrm{~cm} / \mathrm{s}$. PA was passable, stenosis of $30 \%-40 \%$, a magistral-altered reduced $\mathrm{BF}$, LBFB of up to $45 \mathrm{~cm} / \mathrm{s}$. ATA and DPA were passable, a high stenosis, a reduced collateral type BF, LBFV of up to $35 \mathrm{~cm} / \mathrm{s}$. PTA was passable, a high stenosis, a reduced collateral type BF, LBFV of up to $30 \mathrm{~cm} / \mathrm{s}$.

As a result of treatment, there were positive dynamics in the indicators of micro- and macrocirculation, according to CDUS, trophic change regression in the toes, the appearance of a clear demarcation line in the area of necrotic lesion of the toes, an absence of rest pain, and an increase in PWD more than $100 \mathrm{~m}$. Further treatment was continued; necroectomy of the necrotic toes was performed with healing by primary intention. After 4 years, the patient had no complaints. PWD was more than $500 \mathrm{~m}$. 


\section{Conclusions:}

1. An original gene construction based on pl-VEGF165 (a gene therapy drug Neovasculgen) in complex treatment of patients with chronic ischemia and the threat of losing the lower extremities expands the possibilities of successful therapy in inoperable patients. We revealed a significant decrease in the degree of ischemia, pain reduction and relief; an increase in PWD; a regression of trophic disorder; a decrease in the number of amputations; and an increase in the number of LL preservations.

2. The presented method of gene therapy for ChLLI stages II-III can be recommended both as an independent method or in combination with reconstructive vascular surgery or complex conservative treatment to improve longterm results, as well as a preventive therapy for patients with ChLLI stages I-II.

\section{Competing Interests}

The authors declare that they have no competing interests.

\section{References}

1. Bokeriia LA, Eremeeva MV, Arakelyan VS, Demidova OA. Treatment of chronic lower limb ischemia using neoangiogenesis stimulants. Klinicheskaya Fiziologiia Krovoobrashcheniia. 2013;(1):55-60.

2. Savel'ev VS, Koshkin VM, Kunizhev AS. [Critical ischemia resulted from inadequate outpatient management of lower limb chronic arterial obliterations]. Angiol Sosud Khir. 2004;10(1):7-10. [Article in Russian]

3. Cherviakov IuV, Staroverov IN, Nersesian EG, Isaev AA,
Deev RV. [Therapeutic Angiogenesis in Treatment of Patients With Chronic Obliterating Diseases of Lower Limb Arteries]. Angiol Sosud Khir. 2012;18(3):19-27. [Article in Russian]

4. Burger DH, Kappetein AP, Van Bockel JH, Breslau PJ. A prospective randomized trial comparing vein with polytetrafluoroethylene in above-knee femoropopliteal bypass grafting. J Vasc Surg. 2000;32(2):278-283. doi:10.1067/ mva.2000.106496

5. Belch J, Hiatt WR, Baumgartner I, Driver IV, Nikol S, Norgren L, BelleEV; TAMARIS Committees and Investigators. Effect of fibroblast growth factor NV1FGF on amputation and death: a randomised placebo-controlled trial of gene therapy in critical limb ischaemia. Lancet. 2011;377(9781):19291937. doi:10.1016/S0140-6736(11)60394-2

6. Gavrilenko AV, Voronov DA, Konstantinov BA, Bochkov NP. [Combination of Reconstructive Vascular Operations With Gene-Engineering Technologies of Angiogenesis Stimulation: A Present-Day Policy Aimed at Improving the Remote Results of Treating Patients With Lower Limb Chronic Ischaemia]. Angiol Sosud Khir. 2008;14(4):49-53. [Article in Russian].

7. Human Stem Cells Institute: HSCI receives approval to market Neovasculgen - the first Russian gene-therapy drug for treatment of peripheral arterial disease. Available from: http://eng.hsci.ru/news/press-relizy/hsci-receives-approvalto-market-neovasculgen-the-first-russian-gene-therapy-drugfor-treatment-of-peripheral-arterial-disease

8. DeevRV,BozoIY,MzhavanadzeND, VoronovDA, Gavrilenko $\mathrm{AV}$, Chervyakov YuV, et al. pCMV-vegf165 Intramuscular Gene Transfer is an Effective Method of Treatment for Patients With Chronic Lower Limb Ischemia. J Cardiovasc Pharmacol Ther. 2015;20(5):473-482. doi:10.1177/1074248415574336

9. Deev R, Plaksa I, Bozo I, Isaev A. Results of an International Postmarketing Surveillance Study of plVEGF165 Safety and Efficacy in 210 Patients with Peripheral Arterial Disease. Am J Cardiovasc Drugs. 2017;17(3):235242. doi:10.1007/s40256-016-0210-3 